\title{
Prognostic value of the lactate-albumin difference for predicting in- hospital mortality in critically ill patients with sepsis
}

\author{
Gulbin TORE ALTUN ${ }^{1}$ (D), Mustafa Kemal ARSLANTAS² (iD, Pelin CORMAN DINCER ${ }^{3}$ iD, Reyhan ARSLANTAS ${ }^{4}$ (D), Alper KARARMAZ ${ }^{5}$ iD \\ ${ }^{1}$ Anaesthesiology and Reanimation, Memorial Healthcare Group, Sisli, Istanbul, Turkey. \\ ${ }^{2}$ Anaesthesiology and Reanimation, Group Florence Nightingale Hospitals, Istanbul, Turkey. \\ ${ }^{3}$ Department of Oral and Maxillofacial Surgery, School of Dentistry, Marmara University, Istanbul, Turkey. \\ ${ }^{4}$ Anesthesiology and Reanimation, Istanbul Taksim Training and Research Hospital, Istanbul, Turkey. \\ ${ }^{5}$ Department Anesthesiology and Reanimation, School of Medicine, Marmara University, Istanbul, Turkey.
}

Corresponding Author: Gulbin TORE ALTUN

E-mail: dr_gulbin@yahoo.com

Submitted: 02.06.2021 Accepted: 22.10 .2021

\begin{abstract}
Objective: To evaluate the prognostic performance of the lactate-albumin difference (LAD), maximum lactate levels, and the Sequential Organ Failure Assessment (SOFA) score taken on the first day in critically ill patients with sepsis, on in-hospital mortality and 90-day survival.

Patients and Methods: Data from the Medical Information Mart for Intensive Care III (MIMIC III) version 1.4 were evaluated retrospectively. The first day data of adult patients with sepsis diagnosed according to the Third International Consensus Definitions for Sepsis and Septic Shock (Sepsis-3) criteria were recorded. The effectiveness of all diagnostic modalities on admission was analyzed to predict in-hospital mortality and 90-day survival.

Results: In-hospital mortality was $20.3 \%$ among the 2270 patients included in this study. The area below the receiver operating characteristic curve (AUC) value of the LAD was higher than lactate for predicting mortality (AUC, 0.691 ; $95 \%$ confidence interval [CI], 0.663-0.718; $\mathrm{p}<0.01$, and AUC, 0.675; 95\% CI, 0.646-0.703; $\mathrm{p}<0.01$, respectively), and the AUC of SOFA score was the highest (AUC, $0.716 ; 95 \%$ CI, 0.663-0.718). The optimal cutoff of LAD was 0.7.

Conclusion: In addition to its easy and simple calculability relative to the SOFA, the prognostic performance of LAD was superior to maximum lactate levels for predicting in-hospital mortality and 90-day survival in adult patients with sepsis.

Keywords: Lactate-albumin difference, Lactate, Sepsis, In-hospital mortality, Survival
\end{abstract}

\section{INTRODUCTION}

Sepsis is a life-threatening organ dysfunction caused by a dysregulated host response to infection[1]. In sepsis, the primary cause of death is infection, which should be recognized and treated promptly. Based on the Third International Consensus Definitions for Sepsis and Septic Shock (Sepsis-3) definitions, the mortality of patients with sepsis and septic shock in low and middle-income countries is $35.5 \%$ and $75.6 \%$, respectively $[2,3]$. The high mortality rate necessitates using predictive biomarkers to early detection of sepsis and after the efficacy of treatment regimens.

Hypoxia, hypoperfusion, organ dysfunctions, sepsis, and several conditions increase serum lactate levels [4,5]. Inhospital mortality predictors and prognostic indices are used in unselected intensive care unit (ICU) patients and patients with sepsis. The predictive ability of lactate concentration is comparable with the other indices [6,7].

Albumin is one of the negative acute-phase proteins and has an important role in severe sepsis and inflammatory processes; having a low albumin level is associated with increased mortality. Lactate and albumin levels are used independently in predicting in-hospital mortality in sepsis; more studies were conducted using their combination to further increase the predictive value. An increase in lactate and a decrease in albumin levels are associated with high mortality risk; therefore, we hypothesized that lactate-albumin difference (LAD) might be a stronger predictor than these parameters alone. The prognostic performance of the $\mathrm{LAD}$, maximum lactate levels, and initial values of the Sequential Organ Failure Assessment (SOFA)

How to cite this article: Altun Tore G, Arslantas MK, Dincer Corman P, Arslantas R, Kararmaz A. Prognostic value of the lactate-albumin difference for predicting in-hospital mortality in critically ill patients with sepsis. Marmara Med J 2022; 35(1): 61-66. doi: 10.5472/marumj.1059093 
scores [8] on the first admission to ICU for predicting inhospital mortality in patients with sepsis were evaluated.

\section{PATIENTS and METHODS}

In this study, Multiparameter Intelligent Monitoring in Intensive Care III (MIMIC III) version 1.4 data between 2001 and 2012 were retrospectively evaluated. Deidentified data of more than 40,000 ICU patients can be found in the MIMIC III database [9]. The institutional review boards of the Beth Israel Deaconess Medical Center (protocol 2001-P-001699/14) and Massachusetts Institute of Technology (protocol 040.300.0206) approved the use of MIMIC III for research and granted waiver of informed consent.

\section{Cohort Selection}

Data were extracted from MIMIC III using the Structured Query Language scripts [9]. All patients in the MIMIC III were screened, and the first ICU admissions of patients at the age of $\geq 16$ years were selected. Patients at the age of $>89$ years or $>1$ ICU stay; post-cardiac, vascular, or thoracic surgery patients; patients with late diagnoses; patients without available documentation or with missing data; and patients with pregnancy-related diagnoses were excluded.

\section{Statistical Analysis}

Means with standard deviations or medians with interquartile ranges were used to describe patient characteristics. Total numbers and percentages were used to present categorical variables. Continuous variables were analyzed using the Wilcoxon rank-sum tests, and categorical variables were analyzed using Chi-square tests, as appropriate.

Univariable and multivariable logistic regression models were used to analyze the association between in-hospital mortality and the maximum lactate, minimum albumin, and LAD values at admission, and odds ratios were calculated.

We analyzed the capacity of all diagnostic modalities (SOFA, maximum lactate, minimum albumin, and LAD) at admission to predict in-hospital mortality using the area under the receiver operating characteristic curve (AUCs) with 95\% confidence intervals (CIs). The Youden method was used to determine the optimal cutoff value [10]. Furthermore, sensitivity and specificity were determined at this value. The 90-day survival curves from the first day of ICU admission were obtained from Kaplan-Meier estimation.

$\mathrm{P}<0.05$ was considered statistically significant. RStudio (version 1.2.5033) was used for all statistical analyses [11].

\section{RESULTS}

Patients who met the exclusion criteria $(n=40,111)$ of 61532 patients in the ICUs were excluded from the study. After excluding patients with missing and outline initial arterial lactate and venous albumin data $(\mathrm{n}=7424), 3176$ patients were evaluated for the diagnosis of sepsis based on the Sepsis-3 criteria, and finally, 2270 patients were included in the study. They were divided into 2 groups, namely, survived discharged group (survivors group, $\mathrm{n}=1809$ ) and demised in-hospital group (nonsurvivors group, $\mathrm{n}=461$ ). The in-hospital mortality rate $(20.3 \%)$ for the study group is presented in Figure 1.

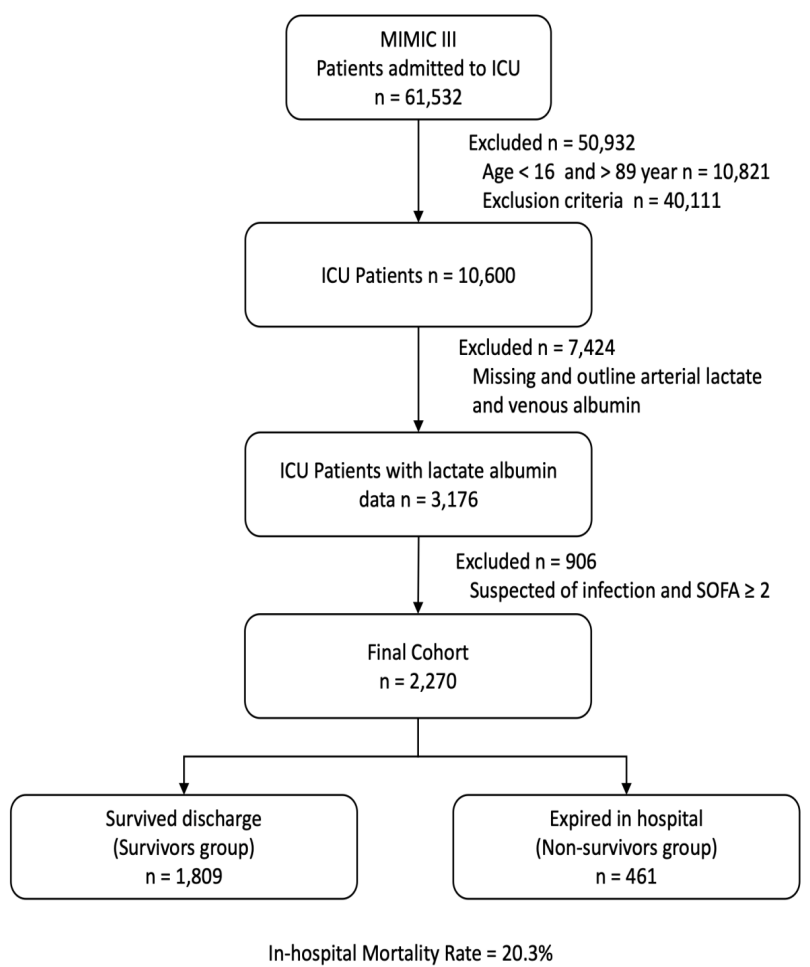

Figure 1. A flowchart of patient inclusion and exclusion

The demographic characteristics of the study cohort are presented in Table I. The nonsurvivors, compared with survivors were older $(\mathrm{p}<0.001)$. SOFA score (first day) $(\mathrm{p}<0.001)$, Elixhauser comorbidity score $(\mathrm{p}<0.001)[12]$, Oxford Acute Severity of Illness Score 
Table I. Patients' baseline demographic characteristics

\begin{tabular}{|c|c|c|c|c|}
\hline Characteristics & $\begin{array}{c}\text { Overall } \\
(\mathbf{n}=2270)^{1}\end{array}$ & $\begin{array}{c}\text { Survivors } \\
(\mathrm{n}=1809 \\
\left.[80 \%]^{1}\right)\end{array}$ & $\begin{array}{l}\text { Nonsurvivors } \\
(\mathrm{n}=461 \\
\left.[20 \%]^{1}\right)\end{array}$ & p value ${ }^{2}$ \\
\hline Age, (y) & $63(52-76)$ & $62(50-74)$ & $69(56-80)$ & $<0.001$ \\
\hline Male, n (\%) & $1312(58 \%)$ & $\begin{array}{l}1039 \\
(57 \%)\end{array}$ & $273(59 \%)$ & 0.5 \\
\hline BMI, (kg/m2) & $28(24-33)$ & $28(24-33)$ & $27(24-32)$ & 0.5 \\
\hline SOFA (first day) & $6(4-8)$ & $5(4-7)$ & $8(5-12)$ & $<0.001$ \\
\hline Admission type,n (\%) & & & & 0.11 \\
\hline Elective & $67(3.0 \%)$ & $60(3.3 \%)$ & $7(1.5 \%)$ & \\
\hline Emergency & $2170(96 \%)$ & $\begin{array}{l}1724 \\
(95 \%) \\
\end{array}$ & $446(97 \%)$ & \\
\hline Urgent & $33(1.5 \%)$ & $25(1.4 \%)$ & $8(1.7 \%)$ & \\
\hline Initially admitted servi & ice, $\mathrm{n}(\%)$ & & & 0.10 \\
\hline CMED & $189(8.3 \%)$ & $\begin{array}{c}143 \\
(7.9 \%) \\
\end{array}$ & $46(10.0 \%)$ & \\
\hline MED & $1459(64 \%)$ & $\begin{array}{c}1157 \\
(64 \%) \\
\end{array}$ & $302(66 \%)$ & \\
\hline NMED & $76(3.3 \%)$ & $60(3.3 \%)$ & $16(3.5 \%)$ & \\
\hline NSURG & $119(5.2 \%)$ & $89(4.9 \%)$ & $30(6.5 \%)$ & \\
\hline SURG & $229(10 \%)$ & $197(11 \%)$ & $32(6.9 \%)$ & \\
\hline TRAUM & $103(4.5 \%)$ & $86(4.8 \%)$ & $17(3.7 \%)$ & \\
\hline Other services & $95(4.2 \%)$ & $77(4.3 \%)$ & $18(3.9 \%)$ & \\
\hline $\begin{array}{l}\text { Elixhauser } \\
\text { comorbidity score }\end{array}$ & $4(0-9)$ & $4(0-9)$ & $7(3-12)$ & $<0.001$ \\
\hline $\begin{array}{l}\text { Mechanical } \\
\text { ventilation, } \mathrm{n}(\%)\end{array}$ & $1271(56 \%)$ & $935(52 \%)$ & $336(73 \%)$ & $<0.001$ \\
\hline Metastatic cancer & $144(6.3 \%)$ & $92(5.1 \%)$ & $52(11 \%)$ & $<0.001$ \\
\hline $\begin{array}{l}\text { Length of hospital } \\
\text { stay, (day) }\end{array}$ & $8(5-14)$ & $9(5-15)$ & $5(2-11)$ & $<0.001$ \\
\hline $\begin{array}{l}\text { Length of ICU stay, } \\
\text { (day) }\end{array}$ & $\begin{array}{c}3.0(1.7- \\
6.7)\end{array}$ & $\begin{array}{c}3.0(1.8- \\
6.3)\end{array}$ & $3.5(1.7-7.2)$ & 0.5 \\
\hline $\begin{array}{l}\text { Lactate }(\max ) \\
(\mathrm{mmol} / \mathrm{L})\end{array}$ & $\begin{array}{c}2.30(1.50- \\
4.00)\end{array}$ & $\begin{array}{c}2.20(1.50- \\
3.50) \\
\end{array}$ & $\begin{array}{c}3.40(2.10- \\
7.00) \\
\end{array}$ & $<0.001$ \\
\hline $\begin{array}{l}\text { Albumin (min) (mg/ } \\
\text { dL) }\end{array}$ & $\begin{array}{c}3.10(2.60- \\
3.60)\end{array}$ & $\begin{array}{l}3.10(2.60- \\
3.60)\end{array}$ & $\begin{array}{c}2.80(2.30- \\
3.30)\end{array}$ & $<0.001$ \\
\hline $\begin{array}{l}\text { Lactate-albumin } \\
\text { difference }\end{array}$ & $\begin{array}{c}-0.70 \\
(-1.60- \\
1.00) \\
\end{array}$ & $\begin{array}{c}-0.90 \\
(-1.70- \\
0.50) \\
\end{array}$ & $\begin{array}{c}0.70(-0.90- \\
4.40)\end{array}$ & $<0.001$ \\
\hline \multicolumn{5}{|l|}{ ICU severity score } \\
\hline OASIS & $35(29-41)$ & $33(28-39)$ & $42(37-47)$ & $<0.001$ \\
\hline APSIII & $48(36-64)$ & $45(34-59)$ & $65(50-84)$ & $<0.001$ \\
\hline SAPSII & $40(32-52)$ & $38(30-48)$ & $55(44-67)$ & $<0.001$ \\
\hline
\end{tabular}

Values are taken within 24 hours of admission to the ICU 1Statistics are presented as median (interquartile range) and number (percentage).2Statistical tests performed were Wilcoxon rank-sum test and Chi-square test of independence. BMI: body mass index, SOFA: Sequential Organ Failure Assessment, CMED: coronary, MED: medical, NMED: neurological, NSURG: neurosurgical, SURG: surgical, TRAUM: trauma, ICU: Intensive Care Unit, OASIS: Oxford Acute Severity of Illness Score, APSIII: Acute Physiology and Chronic Health Evaluation III, SAPSII: Simplified Acute Physiology Score II.

(OASIS) ( $\mathrm{p}<0.001)$, Acute Physiology Score III (APS III) $(\mathrm{p}<$ 0.001), Simplified Acute Physiology Score II (SAPS II) $(\mathrm{p}<$
$0.001)$, and the percentage of mechanical ventilation $\mathrm{p}<0.001)$ and metastatic cancer $(\mathrm{p}<0.001)$ were higher in this group.

Table II. Univariate and multivariate logistic regression analyses of variables potentially associated with in-hospital mortality in intensive care patients

\begin{tabular}{|c|c|c|c|c|c|c|}
\hline \multirow[b]{2}{*}{ Predictor } & \multicolumn{3}{|c|}{$\begin{array}{c}\text { Univariate binary logistic } \\
\text { regression }\end{array}$} & \multicolumn{3}{|c|}{$\begin{array}{c}\text { Multivariate logistic } \\
\text { regression }\end{array}$} \\
\hline & $\mathbf{O R}^{1}$ & $95 \% \mathrm{CI}^{1}$ & $P$-value & $\mathbf{O R}^{1}$ & $95 \% \mathrm{CI}^{1}$ & $P$-value \\
\hline Age & 1.02 & $1.02,1.03$ & $<0.001$ & 1.03 & $1.02,1.04$ & $<0.001$ \\
\hline $\begin{array}{l}\text { Gender }(\mathrm{r} \\
=1)\end{array}$ & 1.08 & $0.87,1.33$ & 0.5 & 0.98 & $0.78,1.25$ & 0.9 \\
\hline SOFA & 1.25 & 1.21, & $<0.0$ & 1.17 & 1.13 & $<0.001$ \\
\hline $\begin{array}{l}\text { Elixhauser } \\
\text { comorbidity } \\
\text { score }\end{array}$ & 1.07 & $1.05,1.08$ & $<0.001$ & 1.02 & $1.01,1.04$ & $<0.00$ \\
\hline Metastatic cancer & 2.37 & $1.65,3.38$ & $<0.001$ & 2.34 & 1.50 & $<0.001$ \\
\hline $\begin{array}{l}\text { Mechanical } \\
\text { ventilation }\end{array}$ & 2.51 & $2.01,3.16$ & $<0.001$ & 1.70 & $1.29,2.25$ & $<0.001$ \\
\hline \multicolumn{7}{|l|}{ Admission type } \\
\hline Emergency & 2.22 & $1.08,5.36$ & 0.048 & 2.04 & $0.89,5.38$ & 0.12 \\
\hline Urgent & 2.74 & $0.89,8.62$ & 0.077 & 1.48 & $0.41,5.43$ & 0.5 \\
\hline \multicolumn{7}{|l|}{ First service } \\
\hline MED & 0.81 & $0.57,1.17$ & 0.2 & 0.76 & $0.50,1.16$ & 0.2 \\
\hline NMED & 0.83 & $0.43,1.55$ & 0.6 & 1.69 & $0.82,3.38$ & 0.14 \\
\hline NSURG & 1.05 & $0.61,1.32$ & 0.9 & 2.08 & $1.14,3.77$ & 0.017 \\
\hline SURG & 0.50 & $0.30,0.83$ & 0.007 & 0.30 & $0.17,0.54$ & $<0.001$ \\
\hline TRAUM & 0.61 & $0.32,1.12$ & 0.12 & 0.75 & $0.36,1.51$ & 0.4 \\
\hline Other & 0.73 & $0.39,1.32$ & 03 & 0.75 & $0.35,1.54$ & 0.4 \\
\hline $\begin{array}{l}\text { Lactate-albumin } \\
\text { difference }\end{array}$ & 1.25 & $1.21,1.29$ & $<0.001$ & 1.36 & $1.13,1.65$ & 0.001 \\
\hline Lactate (max) & 1.25 & $1.20,1.29$ & $<0.001$ & 0.86 & $0.71,1.04$ & 0.12 \\
\hline Albumin (min) & 0.56 & $0 ., 0.45$ & $<0.001$ & - & - & - \\
\hline
\end{tabular}

${ }^{1} \mathrm{OR}$, odds ratio, CI, confidence interval; SOFA, Sequential Organ Failure Assessment; ICU, intensive care unit, BMI: body mass index, CMED: coronary, MED: medical, NMED: neurological, NSURG: neurosurgical, SURG: surgical, TRAUM: trauma, OASIS: Oxford Acute Severity of Illness Score, APSIII: Acute Physiology And Chronic Health Evaluation III, SAPSII: Simplified Acute Physiology Score II.

Values are taken within 24 hours of admission to the ICU.

The P-value of the Hosmer-Lemeshow goodness-of-fit test for the multivariate logistic regression model was 0.286 , and Negelkerke was 0.330 .

Survivors had a longer hospital stay $(\mathrm{p}<0.001)$, but the length of ICU stay $(\mathrm{p}=0.5)$ and admission types $(\mathrm{p}=0.10)$ were similar. Nonsurvivors had higher lactate $\mathrm{p}<0.001)$, higher LAD $(\mathrm{p}<$ $0.001)$, and lower albumin $(\mathrm{p}<0.001)$ levels than survivors. There was no difference between the distribution of the initially admitted service of patients and the groups $(\mathrm{p}=0.10)$.

The analyses of variables potentially associated with in-hospital mortality are presented in Table II. The multivariate logistic regression model identified age $(\mathrm{p}<0.001)$, SOFA score $(\mathrm{p}<$ $0.001)$, Elixhauser comorbidity score $(\mathrm{p}<0.001)$, metastatic cancer $(\mathrm{p}<0.001)$, mechanical ventilation $(\mathrm{p}<0.001)$, initially 
admitted service (Neurosurgery, Surgery) $(\mathrm{p}<0.001)$, and LAD $(\mathrm{p}<0.001)$. The Hosmer-Lemeshow test was not significant $(\mathrm{p}=0.286)$ [ 13], and Negelkerke was 0.330 , indicating that the model adequately fits the data.

The optimal cutoff values, AUC, Youden's index, sensitivity, and specificity for SOFA score, LAD, and maximum lactate are presented in Table II.

The AUC of LAD (AUC, 0.691; 95\% CI, 0.663-0.718) was larger than the maximum lactate (AUC, 0.675; 95\% CI, 0.646-0.703) on the first day (Table III). The AUC of SOFA score was the largest (AUC, 0.716 ; 95\% CI, 0.663-0.718) of these predictors (Table III).

Table III. Cutoff value, Youden index, sensitivity, and specificity of predictors associated with in-hospital mortality

\begin{tabular}{lccccc} 
Predictors & $\begin{array}{c}\text { Cutoff } \\
\text { value }\end{array}$ & AUC & $\begin{array}{c}\text { Youden's } \\
\text { index }\end{array}$ & $\begin{array}{c}\text { Sensitivity } \\
(\%)\end{array}$ & $\begin{array}{c}\text { Specificity } \\
(\%)\end{array}$ \\
\hline SOFA score & 8 & 0.716 & 0.3261 & 57.04 & 75.56 \\
\hline $\begin{array}{l}\text { Lactate-albumin } \\
\text { difference }\end{array}$ & 0.7 & 0.691 & 0.2771 & 50.32 & 77.39 \\
\hline Lactate (max) & 4.4 & 0.675 & 0.4189 & 41.64 & 82.97 \\
\hline
\end{tabular}

SOFA: Sequential Organ Failure Assessment

The Kaplan-Meier 90-day survival curves with risk tables and 95\% CIs of the LAD and SOFA scores on the first day according to the cutoff values are presented in Figure 2. When cutoff values were used, the survival curves indicated a significant difference $(\mathrm{p}<0.001$ for all $)$
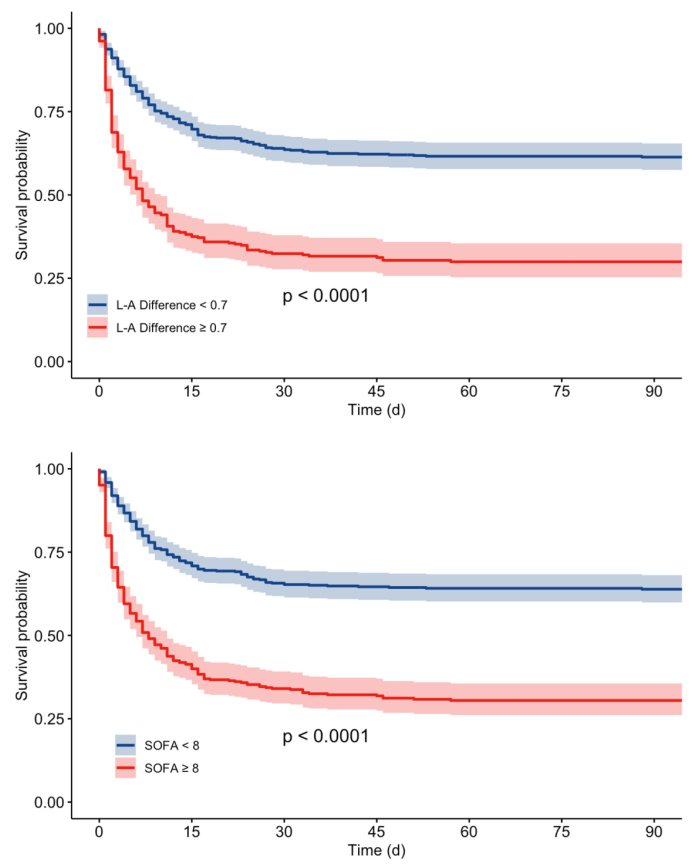

Figure 2. Kaplan-Meier 90-day survival curves with risk tables of the lactate-albumin differences and Sequential Organ Failure Assessment score on the first day according to the cutoff values

\section{DISCUSSION}

In this study, we used the first ICU admission values of critically ill adult patients with sepsis from the MIMIC III version 1.4 to evaluate the prognostic significance of LAD for in-hospital mortality. Compared with maximum lactate in predicting inhospital mortality, LAD had a better prognostic performance in this cohort.

Increased lactate concentrations are strongly correlated with the severity of sepsis and the mortality risk at 30 days and 1 year in patients with septic shock defined by Sepsis-3 [14-16]. Numerous alterations in lactate metabolism such as factors affecting its production, that is, cellular hypoxia, shock, betaadrenergic stimulation, factors affecting its clearance (i.e., liver dysfunction), or simply an intravenous infusion of solutions that contain lactate), may lead to hyperlactatemia [17].

Inflammatory processes may lead to a decline in albumin levels, and the existence of hypoalbuminemia may reflect inflammation. Along with the decrease in total albumin levels, the half-life of albumin is also shortened as the capillary permeability increase enhances the escape of serum albumin. Serum albumin levels are markers of the clinical state, such as malnutrition, various diseases, trauma, or organ failure and result in hypoalbuminemia $[18,19]$. Having excluded exogenous albumin administration, hypoalbuminemia is correlated with increased mortality in sepsis, and in addition to APACHE II, serum albumin levels can be used to predict mortality risk in community-acquired severe sepsis and septic shock $[19,20]$.

In inflammatory states and in sepsis, serum levels of lactate increase, whereas albumin levels decrease. Knowing the actual levels of serum lactate and albumin is important in patients with sepsis, but the importance of using the difference between lactate and serum albumin in providing a variable that positively correlated with organ dysfunction and mortality is unclear. Wang et al. [21] and Lichtenauer et al. [22] studied the correlation of the lactate-albumin ratio (LAR) to organ dysfunction and mortality in patients with sepsis in the ICU and reported a strong association. However, LAD would provide a better correlation than the ratio. Recently, the prognostic value of the LAD over the LAR and maximum lactate in predicting 30-day mortality in critically ill patients was studied [23]. It revealed that LAD is an independent prognostic marker for organ dysfunction and mortality in patients with sepsis. To the best of our knowledge, this study is the first to use LAD from patients with sepsis in the ICU to evaluate in-hospital mortality. Our findings also indicate that LAD is a useful target for predicting 90-day survival in critically ill patients with sepsis; it is superior to a single lactate measurement.

It is known that lactate is important in severe sepsis and septic shock, but it is unclear whether the ratio of lactate and albumin would provide a variable that correlated positively with multiple organ dysfunction syndrome (MODS) and mortality. This study was designed to investigate the prognostic importance of LAR when lactate concentration is $>4 \mathrm{mmol} / \mathrm{L}$.

For in-hospital mortality, Casserly et al., revealed the cutoff value for lactate as $>4 \mathrm{mmol} / \mathrm{L}$ in severe sepsis or septic shock patients, 
with and without hypotension [5]. Our findings indicate that maximum lactate concentration of $>4.4 \mathrm{mmol} / \mathrm{L}$ is a pertinent target in patients with sepsis and septic shock defined by Sepsis-3. The first limitation of this study is that it uses a single institution's database and analyses retrospectively. Secondly, the final cohort may not reflect the entire cohort, owing to missing lactate and albumin data; only $3.6 \%$ of the overall MIMIC III data (2270 of $61,532)$ were evaluated. Finally, only patients with sepsis in the ICU were evaluated, which might have led to selection bias.

Because measuring single lactate and albumin value is simple and inexpensive compared to some of the novel tests, based on our findings that LAD has sufficiently predicted in-hospital mortality, we suggest that it can be used for screening high-risk patients. Further studies are needed for prospective validation and risk stratification.

\section{Conclusion}

The prognostic performance of LAD was superior to that of maximum lactate levels for predicting in-hospital mortality in adult patients with sepsis. In addition to its simplicity and easy calculability, LAD, relative to the SOFA score, is a better prognostic factor than maximum lactate levels. Because this work has evaluated retrospective data and the laboratory values obtained on the first day may affect results, further prospective studies with different predetermined timings of lactate and albumin values are needed on this subject.

\section{Compliance with the Ethical Standards}

Ethical Approval: The institutional review boards of the Beth Israel Deaconess Medical Center (protocol 2001-P001699/14) and Massachusetts Institute of Technology (protocol 040.300.0206) approved the use of MIMIC III for research and granted waiver of informed consent.

Financial Support: The authors have no relevant financial information to close.

Conflict of Interest: The authors have no potential conflicts of interest to disclose

Author Contributions: GTA, MKA, PCD, RA and AK: Conceived and planned the study. They were mainly responsible for the design of the study. MKA and GTA: Collected the data,

MKA and AK: were mainly responsible for analyzing the data, GTA, MKA, PCD and RA: Wrote the first draft of the manuscript. All authors contributed to the interpretation of findings and reviewed the manuscript. GTA, MKA and AK: Reviewed the statistical analyses and made changes to the content of the manuscript. All authors have also provided intellectual contribution to the manuscript.

\section{REFERENCES}

[1] Singer M, Deutschman CS, Seymour CW, et al. The Third International Consensus Definitions for Sepsis and Septic Shock (Sepsis-3). JAMA 2016;315:801-10. doi: 10.1001/ jama.2016.0287.
[2] Vasques F, Duscio E, Romitti F, et al. Septic shock-3 vs 2: an analysis of the ALBIOS

[3] study. Crit Care 2018;22:237. doi: 10.1001/jama.2016.0287.

[4] Baykara N, Akalin H, Arslantas MK, et al. Epidemiology of sepsis in intensive care units in Turkey: a multicenter, point-prevalence study. Crit Care 2018;22:93. doi: 10.1186/ s13054.018.2013-1.

[5] Kraut JA, Madias NE. Lactic acidosis. N Engl J Med 2014;371:2309-19. doi: 10.1056/NEJMra1309483.

[6] Casserly B, Phillips GS, Schorr C, et al. Lactate measurements in sepsis-induced tissue hypoperfusion: results from the Surviving Sepsis Campaign database. Crit Care Med 2015;43:567-73. doi: 10.1097/CCM.000.000.0000000742.

[7] Hayashi Y, Endoh H, Kamimura N, Tamakawa T, Nitta M. Lactate indices as predictors of in-hospital mortality or 90day survival after admission to an intensive care unit in unselected critically ill patients. PLoS One 2020;15:e229135. doi: 10.1371/journal.pone.0229135.

[8] Liu Z, Meng Z, Li Y, et al. Prognostic accuracy of the serum lactate level, the SOFA score and the qSOFA score for mortality among adults with Sepsis. Scand J Trauma Resusc Emerg Med 2019;27:51. doi: 10.1186/s13049.019.0609-3

[9] Vincent JL, Moreno R, Takala J, et al. The SOFA (Sepsisrelated Organ Failure Assessment) score to describe organ dysfunction/failure. On behalf of the Working Group on Sepsis-Related Problems of the European Society of Intensive Care Medicine. Intensive Care Med 1996;22:707-10.

[10] Johnson AE, Pollard TJ, Shen L, et al. MIMIC-III, a freely accessible critical care database. Sci Data 2016;3:160035. doi: 10.1038/sdata.2016.35.

[11] Fluss R, Faraggi D, Reiser B. Estimation of the Youden Index and its associated cutoff point. Biom J 2005;47:458-72.

[12] Team R. RStudio: Integrated Development for R. RStudio: PBC, Boston, MA, 2020. Available from: http://www.rstudio. com/ Accessed on: 23.08.2021

[13] Elixhauser A, Steiner C, Harris DR, Coffey RM. Comorbidity measures for use with administrative data. Med Care 1998;36:8-27.

[14] Hosmer DW, Lemeshow S. Applied Logistic Regression. Second ed. New York: Wiley, 2000.

[15] Villar J, Short JH, Lighthall G. Lactate predicts both short - and long-term mortality in patients with and without sepsis. Infect Dis (Auckl) 2019;12:117.863.3719862776. doi: 10.1177/117.863.3719862776.

[16] Ryoo SM, Lee J, Lee YS, et al. Lactate level versus lactate clearance for predicting mortality in patients with septic shock defined by Sepsis-3. Crit Care Med 2018;46:e489-e95. doi: 10.1097/CCM.000.000.0000003030.

[17] Gattinoni L, Vasques F, Camporota L, et al. Understanding lactatemia in human sepsis. potential impact for early management. Am J Respir Crit Care Med 2019;200:582-9. doi: 10.1164/rccm.201.812.2342OC.

[18] Vincent JL, Quintairos ESA, Couto L, Jr., Taccone FS. The value of blood lactate kinetics in critically ill patients: a systematic 
review. Crit Care 2016;20:257. doi: 10.1186/s13054.016.14035.

[19] Soeters PB, Wolfe RR, Shenkin A. Hypoalbuminemia: Pathogenesis and clinical significance. JPEN J Parenter Enteral Nutr 2019;43:181-93. doi: 10.1002/jpen.1451.

[20] Yin M, Si L, Qin W, Li C, Zhang J, Yang H, et al. Predictive value of serum albumin level for the prognosis of severe sepsis without exogenous human albumin administration: a prospective cohort study. J Intensive Care Med 2018;33:68794. doi: 10.1177/088.506.6616685300

[21] Artero A, Zaragoza R, Camarena JJ, Sancho S, Gonzalez R, Nogueira JM. Prognostic factors of mortality in patients with community-acquired bloodstream infection with severe sepsis and septic shock. J Crit Care 2010;25:276-81. doi: 10.1016/j. jcrc.2009.12.004

[22] Wang B, Chen G, Cao Y, Xue J, Li J, Wu Y. Correlation of lactate/albumin ratio level to organ failure and mortality in severe sepsis and septic shock. J Crit Care 2015;30:271-5. doi: 10.1016/j.jcrc.2014.10.030

[23] Lichtenauer M, Wernly B, Ohnewein B, et al. The lactate/ albumin ratio: a valuable tool for risk stratification in septic patients admitted to icu. Int J Mol Sci 2017;18:1893. doi: 10.3390/ijms18091893.

[24] Arslantas MK, Arslantas R, Dincer PC, Altun GT, Kararmaz A. Prognostic value of the lactate-albumin difference in predicting 30-day mortality in critically ill patients. Shock 2021;56:150-1. doi: 10.1097/SHK.000.000.0000001613. 